\title{
Decodificación y comprensión lectora en escolares con Trastorno Específico del Lenguaje
}

\section{Decoding and reading comprehension in children with specific language impairment}

Carmen Julia Coloma

Departamento de Fonoaudiología Centro de Investigación Avanzada en

Educación (CIAE) Universidad de Chile

Valentina Caroca Universidad de Chile

Andrea Kurte Universidad de Chile

Bernardita Melipil

Universidad de Chile

Geraldine Ortiz

Universidad de Chile

Camilo Quezada

Departamento de Fonoaudiología Universidad de Chile

\section{RESUMEN}

El objetivo del presente estudio fue describir el desempeño en decodificación y en comprensión lectora de escolares con Trastorno Específico del Lenguaje (TEL). Para ello, se trabajó con 58 niños de 2 do básico, distribuidos en dos grupos: uno de 29 escolares con Trastorno Específico del Lenguaje (TEL) y otro de 29 escolares con desarrollo típico (DT). Las habilidades evaluadas fueron decodificación y comprensión lectora. Los resultados generales mostraron que el grupo con TEL presentó un menor desempeño que los niños con DT en ambas habilidades lectoras. Los menores con TEL tendieron a mostrar un mejor rendimiento en decodificación que en comprensión lectora. Respecto a la decodificación, los resultados mostraron que los niños con TEL lograban decodificar palabras comunes y trisilábicas. En cuanto a comprensión lectora, comprendían frases, oraciones y textos. Sin embargo, en el nivel del texto evidenciaron dificultades para responder las preguntas inferenciales y en el reconocimiento de la idea central.

Palabras clave: decodificación, comprensión lectora, Trastorno Específico del Lenguaje.

\begin{abstract}
This study was conducted to describe the reading performance of schoolers with Specific Language Impairment (SLI), observing their decoding skills and reading comprehension. A sample of 58 children of a second-grade primary school was studied, evenly grouped in two conditions: SLI and TLD (Typical Language Development). General results show that children with SLI perform lower than children with TLD on decoding and reading comprehension. Results for decoding skills showed that SLI children were able to decode trisyllabic words and common words. As for reading comprehension abilities, children were able to understand phrases, complete sentences, and give a proper title to the texts they read.
\end{abstract}

Keywords: decoding, reading comprehension, Specific Language Impairment.

Contacto con el autor: Carmen Julia Coloma T. Santiago - Chile Correo-e: carmenjuliacoloma@gmail.com

Recibido: $15 / 08 / 2018$ Aceptado: 19/10/2018 


\section{Introducción}

El Trastorno Específico del Lenguaje (TEL) corresponde a una limitación importante en la capacidad lingüística que afecta a algunos niños. Dicha limitación no se explica por pérdida auditiva, daño cerebral, baja inteligencia, déficit motor o deprivación sociocultural (Aguado, 2004; Fresneda \& Mendoza, 2005).

Las características lingüísticas de los niños con TEL son variadas y dependen de la lengua que hable el menor (Leonard, 2009). En cuanto a las principales dificultades lingüísticas, se advierten fundamentalmente problemas en los planos fonológico, morfosintáctico, léxico y discursivo (Coloma et al., 2012; Hulme \& Snowling, 2011; Ramus, Marshall, Rosen, \& van der Lely, 2013).

Debido a sus dificultades lingüísticas, los niños con TEL son un grupo de riesgo para el aprendizaje lector (Botting, Simkin, \& Conti-Ramsden, 2006). Al respecto, se ha demostrado que la persistencia de las dificultades lingüísticas repercuten en las habilidades lectoras, incluso hasta la adolescencia (Bishop \& Adams, 1990; Catts, Fey, Tomblin, \& Zhang, 2002; Catts, Bridges, Little, \& Tomblin, 2008).

Los niños con dificultades lectoras se pueden clasificar a en distintos grupos: a) disléxicos, que presentan bajo desempeño en decodificación, pero buen desempeño en comprensión oral; b) pobres comprendedores, que muestran una decodificación adecuada pero una comprensión oral deficitaria y c) mixtos, que manifiestan problemas en decodificación y en comprensión oral (Hoover \& Gough, 1990). Según Bishop \& Snowling (2004), el comportamiento lector de los niños con TEL corresponde al grupo de niños mixtos. Ello debido a que el cuadro clásico de TEL implica problemas en el procesamiento fonológico y en las habilidades semánticas, sintácticas y discursivas. Así, las dificultades fonológicas impactarían en la decodificación, mientras que los problemas semánticos, sintácticos y discursivos afectarían la comprensión lectora.

La mantención de la dificultad lectora en los niños con TEL ha sido reportada en lenguas opacas, como el inglés (Catts et al., 2008) y en idiomas cuya ortografía es transparente, como el italiano (Brizzolara et al., 2011). Así, en escolares con TEL angloparlantes se observó que sus desempeños en decodificación y en comprensión lectora son inferiores al de sus controles, en segundo grado. Sin embargo, en grados superiores ellos incrementaron sus rendimientos en estas habilidades lectoras. Pese a su mejoría, no lograron igualarse a los niños con desarrollo típico (Catts et al., 2008). En otro estudio en que participaron jóvenes italianos con antecedentes de TEL, se determinó que mantenían problemas en decodificación y en comprensión lectora incluso en la adolescencia (Brizzolara et al., 2011).

A pesar de que los problemas se producen tanto en decodificación como en comprensión lectora, varios estudios han mostrado que los menores con TEL tienden a manifestar más dificultades en comprensión lectora que en decodificación (Bishop, McDonald, Bird, \& Hayiou-Thomas, 2009; Catts et al., 2002; Kelso, Fletcher, \& Lee, 2007).

Existen diversos trabajos en esta área, aunque la mayoría se ha realizado con niños que hablan inglés (Bishop \& Adams, 1990; Catts et al., 2002; Catts et al., 2008; Kelso et al., 2007). Como ya se 
mencionó, el inglés es una lengua que se caracteriza por poseer una ortografía opaca, por lo tanto, los desempeños en lectura de los sujetos angloparlantes no se pueden homologar a los rendimientos lectores que pueden evidenciar hablantes de una lengua transparente, como es el español. Lo anterior debido a que el tipo de ortografía puede afectar el desempeño en decodificación, ya que en esta habilidad lectora es fundamental la asociación fonema-grafema. Las ortografías opacas se caracterizan por la inconsistencia en la relación fonema-grafema, lo que implica que un fonema puede ser representado ortográficamente de varias maneras (Diuk \& Ferroni, 2013). Al contrario, en la ortografía transparente la relación fonema-grafema tiende a ser consistente (Carrillo \& Alegría, 2009).

Los estudios en lenguas cuyas ortografías son transparentes son más escasos. Además, la mayoría de ellos se centra en la relación entre las habilidades lingüísticas y la lectura en niños con TEL (Acosta, Moreno, \& Axpe, 2010; V. Acosta, Ramírez, Del Valle, \& de Castro, 2016; Brizzolara, Gasperini, Pfanner, Cristofani, Casalini,\& Chilosi, 2011; Buil-Legaz, Aguilar-Mediavilla, \& Rodríguez-Ferreiro, 2015). En consecuencia, la caracterización de la decodificación y de la comprensión lectora de los niños hispanohablantes con TEL se ha estudiado menos.

El conocimiento sobre los problemas lectores de los escolares hispanohablantes con TEL puede enriquecerse al caracterizar su desempeño lector, ya que se entrega información específica sobre su lectura.

El objetivo de este trabajo es describir los desempeños en decodificación y en comprensión lectora de niños hispanohablantes con TEL.

\section{Metodología}

\section{Participantes}

En esta investigación participaron un total de 58 alumnos de $2^{\circ}$ básico que asistían a escuelas con Proyectos de Integración Escolar (PIE), pertenecientes a la Región Metropolitana de Chile. Los establecimientos educacionales con PIE posibilitan que los niños con TEL estén en aula regular. Además, se les ofrecen apoyos específicos relacionados con ámbitos lingüísticos, cognitivos y sociales.

Los participantes fueron divididos en dos grupos: el primero estaba compuesto por 29 niños con TEL (14 mujeres y 15 hombres) y el otro estaba formado por 29 escolares con desarrollo típico (DT) (12 mujeres y 17 hombres). El promedio de edad del grupo de los niños con TEL fue 8 años y el promedio de edad del grupo control fue 7 años, 11 meses.

El diagnóstico de los escolares con TEL lo realizó el profesional fonoaudiólogo del establecimiento escolar. Para el diagnóstico utilizó los criterios estándares exigidos por el Ministerio de Educación (2010). Dichos criterios exigen que el niño muestre rendimientos disminuidos en al menos dos de las pruebas que miden expresión y/o comprensión del lenguaje, para ser diagnosticado con TEL. Las pruebas para evaluar expresión abordan los componentes fonológicos (Pavez, Maggiolo, \& Coloma, 2008) y gramatical (Pavez, 2003). A su vez, las que evalúan la comprensión miden gramática (Pavez, 2003) y vocabulario (Pavez, 2004). Es importante señalar que los niños con TEL recibían apoyo fonoaudiológico desde $1^{\circ}$ básico, es decir, 
llevaban 2 años de tratamiento.

Los niños del grupo control presentaban desarrollo típico y eran compañeros de curso de los escolares con TEL. El requisito para ingresar a este grupo era la ausencia de antecedentes de problemas de lenguaje o de aprendizaje. Para obtener la información se entrevistó a la profesora de los escolares. En consecuencia, el grupo control se pareó por edad y nivel escolar. Igualar por nivel escolar era relevante, ya que era necesario que todos tuvieran una experiencia de enseñanza lectora equivalente.

Todos los niños fueron evaluados tanto en habilidades cognitivas no verbales como en habilidades auditivas. Estas evaluaciones buscaban asegurar que los participantes se ubicaban dentro de rangos normales en ambas habilidades. Las habilidades cognitivas se evaluaron con el Test de Matrices Progresivas, Escala Coloreada para Evaluar las Habilidades cognitivas (Raven, Court, \& Raven, 2005) y las auditivas mediante una audiometría de barrido realizada con un audiómetro clínico marca Interacoustic modelo AD629 en las frecuencias 500, 1000, 2000 y $4000 \mathrm{~Hz}$ (American Speech Language Hearing Association Commitee on language, Speach and Hearing Services in the Schools, 1980).

En síntesis, los niños del grupo en estudio presentaban diagnóstico de TEL y evidenciaban habilidades cognitivas no verbales $y$ auditivas apropiadas para su edad. Por su parte, el grupo control manifestaba un desarrollo apropiado tanto en lenguaje como en habilidades auditivas y cognitivas no verbales.

\section{Instrumentos}

La decodificación se evaluó mediante la subprueba Identificación de letras y palabras de la batería Language Survey-Revised (Versión española) de Woodcock, Muñoz, Ruef, \& Alvarado (2005).

La comprensión lectora se estudió mediante dos instrumentos: la subprueba Comprensión de textos de la batería Language Survey-Revised (Versión español) de (Woodcock et al., 2005) y la subprueba de Comprensión de textos del Test de Lectura y Escritura en Español de Defior et al. (2006). En este trabajo, la subprueba Comprensión de textos se identificó como CL1 y la subprueba Comprensión de textos se consignó como CL2.

A continuación, se describen las subpruebas mencionadas:

1. Identificación de letras y palabras. Está constituida por 75 ítems graduados por dificultad. Los primeros ítems requieren que el niño identifique las letras del alfabeto, para luego leer palabras de complejidad fonológica creciente. A cada respuesta correcta se le asigna 1 punto, por lo que la puntuación máxima es de 75 . La confiabilidad de la prueba es de 0.96, según la subprueba de Kuder y Richardson.

2. CL1. Está compuesta por 32 ítems. En los ítems iniciales el niño debe hacer coincidir símbolos de diferentes objetos con sus respectivos dibujos. En los siguientes, debe seleccionar el dibujo correcto y asociarlo a una frase escrita. Posteriormente, el niño lee oraciones que debe completar con una palabra. Finalmente, lee breves discursos expositivos que aumentan en longitud, sintaxis y léxico. En dichas exposiciones faltan palabras que el niño debe 
completar para dar sentido al texto. Al igual que en la subprueba de decodificación, se asigna 1 punto por cada respuesta correcta y la máxima puntuación que se puede alcanzar es 32 puntos. Su confiabilidad, según la subprueba de Kuder y Richardson es de 0.95 .

3. CL2. Se compone de 24 ítems basados en la lectura de dos textos narrativos y uno expositivo. Luego de leer cada texto el niño debe responder tres preguntas literales y tres inferenciales. Además, debe seleccionar el título y el resumen más adecuado. El título está centrado en el reconocimiento del personaje principal y en su atributo, mientras que el resumen está focalizado en la identificación de las ideas principal y secundaria. A las preguntas literales correctas se le asignan 2 puntos, 1 punto si está incompleta y 0 si es incorrecta. A las inferenciales se las valora con 2 puntos si están correctas y 0 si están incorrectas. A cada respuesta correcta del ítem referido al título se le asigna 2 puntos. Finalmente, a la pregunta de resumen se le otorga 2 puntos si apunta a la idea principal y secundaria, 1 punto si solo indica la idea secundaria y 0 si responde con una idea externa. La puntuación máxima por texto es de 16 puntos y la de la subprueba es de 48 puntos. La subprueba tiene un alfa de Cronbach de 0.69 .

\section{Procedimientos}

La evaluación la efectuaron fonoaudiólogas y profesoras capacitadas especialmente para este fin. Dicha evaluación se realizó individualmente en el establecimiento educacional, durante la jornada escolar, en el segundo semestre del año. Los instrumentos se administraron en dos sesiones: en la primera se aplicaron las subpruebas Identificación de letras y palabras y CL1. En la segunda sesión se administró la subprueba CL2.

\section{Resultados}

Los resultados que se exponen corresponden a las comparaciones entre ambos grupos, los desempeños lectores de los participantes y la clasificación de los niños, según su rendimiento lector.

La comparación de la decodificación y de la comprensión lectora se efectuó mediante la prueba t de Student. Se convirtieron los puntajes brutos obtenidos por los niños de cada instrumento a porcentajes basados en el puntaje máximo de cada subprueba. Esta conversión permitió traspasar a una misma escala las diferentes puntuaciones de las subpruebas.

La tabla 1 y el gráfico 1 muestran los resultados de la comparación entre ambos grupos. Los resultados señalan que los promedios del grupo de niños con TEL son significativamente más bajos en todas las subpruebas que los promedios del grupo control.

El gráfico 1 revela que los rendimientos de los niños con TEL son inferiores en las tres subpruebas a los de los niños con DT, aunque muestran trayectorias similares. Además, se puede observar que el rendimiento de los niños con TEL en la subprueba Identificación de letras y palabras está algo más cerca al desempeño de los niños con DT. Este fenómeno no se advierte en las pruebas de comprensión. 
El análisis descriptivo en las subpruebas Identificación de Letras y Palabras, CL1 y CL2 implicó determinar niveles de desempeño. En Identificación de letras y palabras y CL1 se determinaron niveles a partir del aumento de la dificultad de la tarea (ver tablas 2 y 3). Se ubicó a los participantes en el nivel más alto cuando respondieran de manera correcta al menos el $80 \%$ de las preguntas. Por ejemplo, un menor que responde el $80 \%$ del nivel "Señalización de monosílabos y bisílabos" y luego contesta menos de ese porcentaje en los siguientes niveles se ubica en el nivel señalado. En el caso de que el sujeto en varios niveles responda el $80 \%$ de los ítems, se ubica en el nivel más alto del instrumento.

Tabla 1

Comparación del desempeño lector en decodificación y en comprensión lectora entre los niños con TEL y los niños con DT.

\begin{tabular}{|c|c|c|c|c|c|c|c|c|}
\hline Subprueba & Grupo & $\mathrm{N}$ & Media [\%] & DS [\%] & Min [\%] & Máx [\%] & $t$ & $p$ \\
\hline \multirow{2}{*}{$\begin{array}{l}\text { Identificación de } \\
\text { letras y palabras }\end{array}$} & DT & 29 & 52,09 & 9,73 & 41,33 & 100 & \multirow{2}{*}{3,52} & \multirow{2}{*}{$<0,001$} \\
\hline & TEL & 29 & 42,25 & 11,47 & 25,33 & 52 & & \\
\hline \multirow{3}{*}{ CL1 } & DT & 29 & 46,44 & 10,29 & 18,75 & 53,12 & \multirow{3}{*}{4,50} & \multirow{3}{*}{$<0,001$} \\
\hline & & & & & & & & \\
\hline & TEL & 29 & 30,06 & 16,67 & 9,38 & 53,12 & & \\
\hline \multirow{3}{*}{$\mathrm{CL} 2$} & DT & 29 & 76,01 & 9,48 & 56,25 & 93,75 & \multirow{3}{*}{5,43} & \multirow{3}{*}{$<0,001$} \\
\hline & & & & & & & & \\
\hline & TEL & 29 & 54,31 & 19,3 & 12,5 & 81,25 & & \\
\hline
\end{tabular}

* Diferencias es significativa si $p<0,001$

80

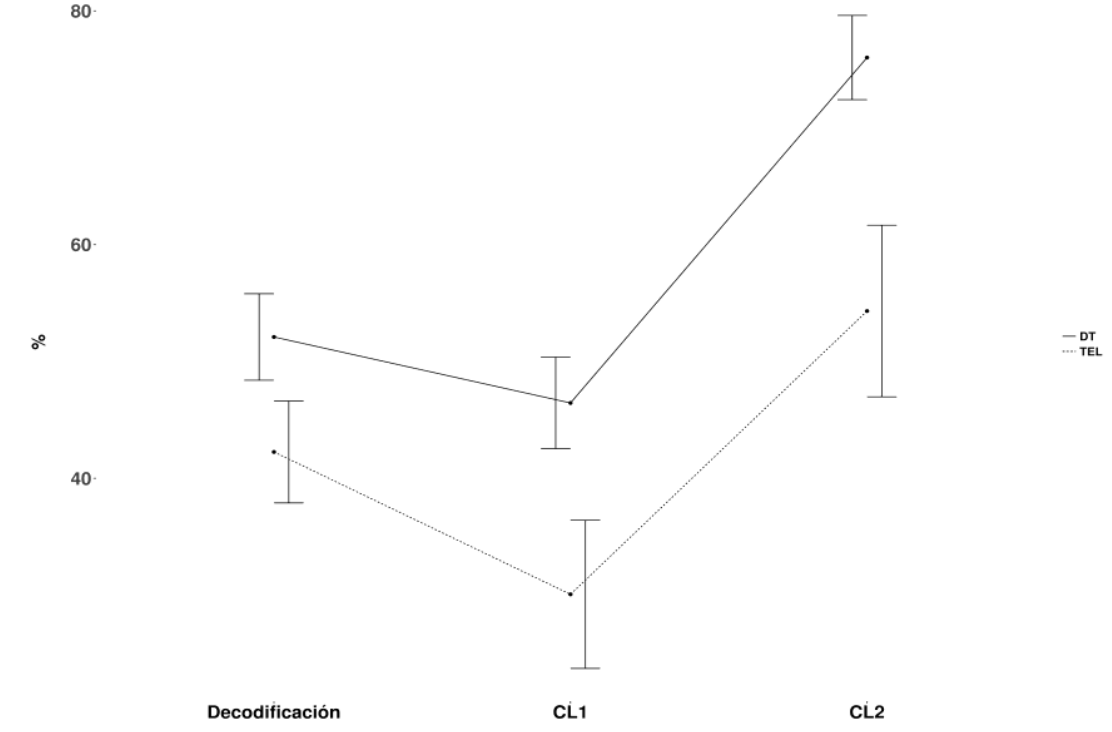

Gráfico 1. Comparación de las medias en porcentaje entre grupos TEL y DT, por cada subprueba aplicada. Las barras de error representan intervalos de confianza de $95 \%$. 
Tabla 2

Porcentaje de niños que se ubica en cada nivel de la subprueba de Identificación de letras y palabras.

\begin{tabular}{lcc}
\hline $\begin{array}{l}\text { Niveles de las subpruebas de identificación de letras y } \\
\text { palabras }\end{array}$ & Grupo TEL & Grupo DT \\
\hline Identificación de letras & $0 \%$ & $0 \%$ \\
Señalización de monosílabos y bisílabos & $27,58 \%$ & $10,34 \%$ \\
Decodificación de monosílabos y bisílabos & $13,79 \%$ & $86,20 \%$ \\
$\begin{array}{l}\text { Decodificación de palabras trisilábicas y comunes } \\
\begin{array}{l}\text { Decodificación de palabras multisilábicas complejas } \\
\text { fonológicamente y poco frecuentes }\end{array}\end{array}$ & $58,62 \%$ & $3,44 \%$ \\
\hline
\end{tabular}

La tabla 2 expone los distintos niveles que alcanzaron los niños en la subprueba de Identificación de letras y palabras. Para ello, se informa el porcentaje de niños ubicado en cada nivel, según el desempeño que obtuvieron en esta subprueba.

En la tabla 2 se observa que ambos grupos se encuentran mayoritariamente en el nivel de decodificación de palabras trisilábicas y comunes. Sin embargo, pese a que los niños con TEL se ubican principalmente en dicho nivel, un $41,37 \%$ de ellos se sitúa en niveles inferiores, en cambio, solo un $10,3 \%$ de los menores con DT están en un nivel inferior.

Es destacable que el peor desempeño del grupo con TEL corresponde a señalar palabras monosilábicas y bisilábicas. Por su parte, el rendimiento más disminuido del grupo control corresponde a decodificar palabras monosilábicas y bisilábicas.

La tabla 3 muestra el desempeño de ambos grupos en los distintos niveles de la subprueba CL1, representado mediante el porcentaje de niños que se ubica en cada nivel.

Tabla 3

Porcentaje de niños que se ubica en cada nivel de la subprueba CL1.

\begin{tabular}{lcc}
\hline Niveles de la subprueba CL1 & Grupo TEL & Grupo DT \\
\hline Señalización de objetos (palabras aisladas) & $10,34 \%$ & $0 \%$ \\
Lectura de frase & $48,27 \%$ & $13,79 \%$ \\
Lectura y completación de Oraciones & $41,37 \%$ & $86,20 \%$ \\
Lectura y completación de Párrafo Expositivo & $0 \%$ & $0 \%$ \\
\hline
\end{tabular}

Se puede observar en la tabla 3 que la mayoría de los niños con TEL se distribuyen tanto en el nivel de lectura de frase como el de lectura de oraciones. A su vez, la mayor parte del grupo control logra leer oraciones.

En la tabla 4 se muestran los niveles de la subprueba CL2 y el porcentaje de niños que se ubica en cada uno de ellos. Se dividió el puntaje máximo 
de cada subprueba en 3, lo que permitió obtener niveles con diferentes rangos de puntajes. Así, se identificaron 3 niveles: bajo, regular y óptimo.

La tabla 4 muestra que la mayoría de los niños con TEL $(75,86 \%)$ puede identificar el título correcto. A su vez, un disminuido porcentaje (24,13\%) es capaz de responder a las preguntas inferenciales. En cambio, el mayor porcentaje del grupo control puede responder preguntas literales $(89,65 \%)$, reconocer el título $(96,55 \%)$ e identificar el resumen $(82,75 \%)$ correcto a cada texto.

Tabla 4

Porcentaje de niños que se ubica en cada nivel de la subprueba CL2.

\begin{tabular}{|c|c|c|c|}
\hline Niveles & Rangos de puntaje & Grupo TEL & Grupo DT \\
\hline \multirow{3}{*}{ Preguntas Literales } & {$[0-5]$} & $17,24 \%$ & $0 \%$ \\
\hline & {$[6-11]$} & $37,93 \%$ & $10,35 \%$ \\
\hline & [12-18] & $44,82 \%$ & $89,65 \%$ \\
\hline \multirow{3}{*}{ Preguntas Inferenciales } & {$[0-5]$} & $27,58 \%$ & $0 \%$ \\
\hline & {$[6-11]$} & $48,27 \%$ & $51,72 \%$ \\
\hline & [12-18] & $24,13 \%$ & $48,27 \%$ \\
\hline \multirow{3}{*}{ Reconocimiento del Título } & {$[0-1]$} & $6,89 \%$ & $0 \%$ \\
\hline & {$[2-3]$} & $17,24 \%$ & $3,44 \%$ \\
\hline & {$[4-6]$} & $75,86 \%$ & $96,55 \%$ \\
\hline \multirow{3}{*}{ Identificación del Resumen } & {$[0-1]$} & $17,24 \%$ & $0 \%$ \\
\hline & {$[2-3]$} & $44,82 \%$ & $17,24 \%$ \\
\hline & {$[4-6]$} & $37,93 \%$ & $82,75 \%$ \\
\hline
\end{tabular}

Es importante señalar que en ambos grupos los porcentajes de participantes que logran un rendimiento óptimo en las preguntas inferenciales es pequeño. Ello sugiere que es la tarea más difícil de esta prueba.

Finalmente, se clasificaron los niños según sus desempeños lectores. Para ello, se tomó como referencia el promedio de rendimiento de los niños con DT en cada subprueba. A este promedio, se le restó 1,5 unidades de desviación estándar del grupo DT en cada variable. El resultado de esta operación determinó un puntaje de corte que se usó para clasificar los puntajes obtenidos por todos los niños en decodificación y en comprensión lectora. Un puntaje superior al punto de corte fue considerado como "Sin dificultades". Un puntaje igual inferior, en cambio, fue considerado como indicador de dificultades. Por último, cabe señalar que, en el caso de la comprensión lectora, un niño debía estar bajo el punto de corte en las dos subpruebas de lectura para quedar clasificado como niño con dificultades lectoras. 
El gráfico 2 muestra la distribución de los niños

DT.

con y sin dificultades lectoras de los grupos con TEL y

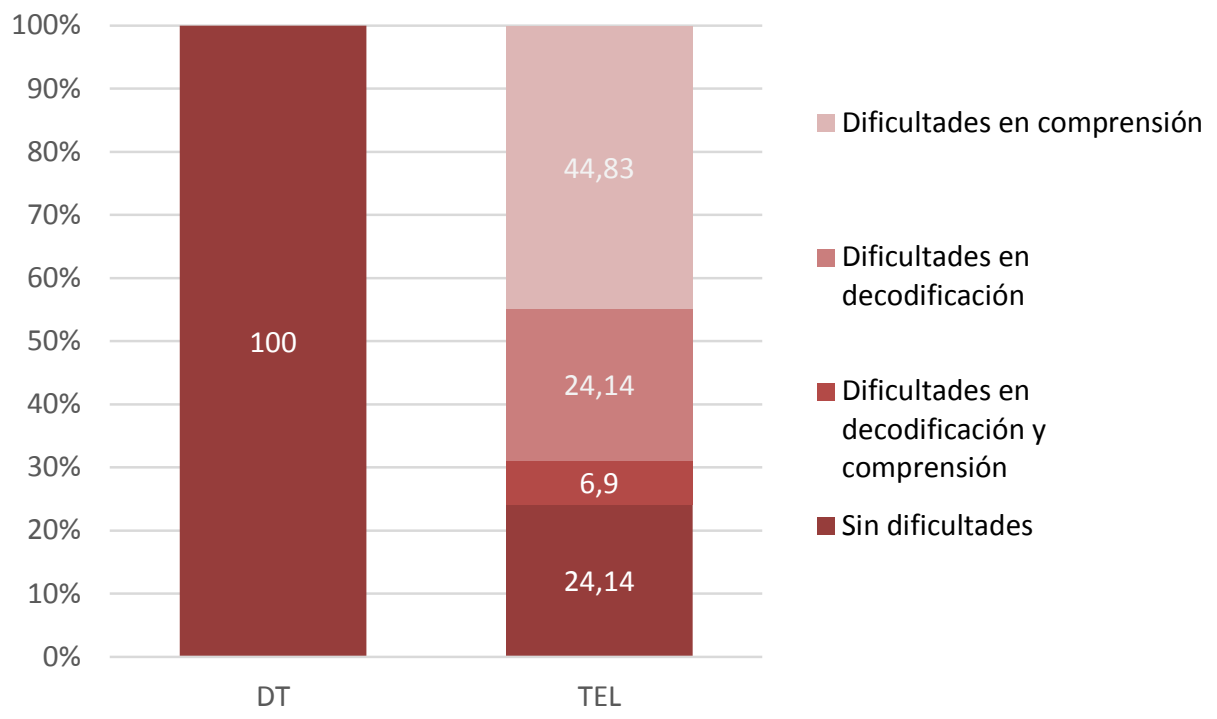

Gráfico 2. Distribución de los niños con y sin dificultades lectoras

El gráfico 2 muestra que un poco más del $50 \%$ de los niños con TEL presentan dificultades. Además, se advierte que las dificultades en comprensión lectora son predominantes en ellos. La cantidad de niños que solo presenta dificultades en decodificación es muy baja y representa solo el 6,9\% de los niños TEL. Por su parte, ningún niño con DT presenta dificultades en lectura.

\section{Discusión}

El presente trabajo analiza y discute los resultados en los siguientes niveles: 1) comparaciones de los desempeños en lectura, 2) características de la decodificación y la comprensión lectora en los niños con TEL, 3) grado de dificultad de la decodificación y de la comprensión lectora de los niños con TEL y 4) descripción del perfil lector de los niños con TEL.
Los resultados referidos al nivel 1 muestran que los niños con TEL obtienen medias significativamente menores en relación a los niños con DT, tanto en decodificación como en comprensión lectora. Estos hallazgos coinciden con lo expuesto en otros estudios que señalan que estos niños presentan un rendimiento lector inferior al de sus controles (Catts et al., 2008; Coloma, Sotomayor, De Barbieri, \& Silva, 2015).

Los datos correspondientes al nivel 2 se pueden comparar con los obtenidos por Tapia (2016), ya que también se trabajó con niños chilenos que eran muy similares a los del presente estudio. Además, la decodificación y la comprensión lectora se evaluó con dos de los instrumentos utilizados en la actual investigación, que corresponden a las subpruebas de la batería Language Survey-Revised (Versión española) de Woodcock et al. (2005).

En cuanto a la decodificación, la mayoría de los 
niños con TEL de $2^{\circ}$ básico decodifican palabras comunes y trisilábicas. Al relacionar estos datos con los obtenidos por Tapia (2016), se advierte que progresan entre primero y segundo básico, ya que transitan desde la identificación de letras a la decodificación de palabras. Sin embargo, dicho progreso no les permite alcanzar el mismo nivel de sus compañeros con DT, lo que sugiere un avance más lento y dificultoso. La lentitud en el aprendizaje de la decodificación podría deberse a que las representaciones fonológicas de los niños con TEL tienden a ser globales y escasamente diferenciadas (Acosta et al., 2016). Ello afectaría su desempeño en conciencia fonológica, lo que a su vez impactaría negativamente en el aprendizaje de la decodificación. Las dificultades en conciencia fonológica en los niños con TEL se han constatado en trabajos previos (Acosta, Moreno, \& Axpe, 2011; De Barbieri \& Coloma, 2004; Nicolielo \& Rocha de Vasconcellos, 2014) y el impacto de esta habilidad metalingüística en decodificación también ha sido corroborado (Bravo, Villalón, \& Orellana, 2002; Ehri et al., 2001).

Como ya se mencionó, la comprensión lectora se midió mediante dos instrumentos, a fin de abordar diferentes aspectos de esta habilidad lectora. Los resultados de la subprueba CL1 muestran que prácticamente la mitad de los niños con TEL alcanza el nivel de lectura que implica leer oraciones incompletas y completarlas con una palabra para darle sentido. Por su parte, los hallazgos de Tapia (2016) indican que en primero básico la mayoría de los niños con TEL solo pueden relacionar símbolos que representan dibujos de objetos. Por lo tanto, los escolares con TEL avanzan entre primero y segundo básico en comprensión lectora, tal como ocurre con la decodificación. Sin embargo, al compararlos con el grupo control sus rendimientos se mantienen descendidos en segundo básico.

Resultados similares encontraron Catts et al. (2008), ya que mostraron que los niños con TEL incrementaron sus rendimientos en decodificación y en comprensión lectora desde segundo grado hasta décimo grado. Sin embargo, a pesar de su evolución no alcanzaron los rendimientos lectores de sus controles.

Al analizar las diferentes tareas de la subprueba $C L 2$, se advierte que la tarea que menos dificultades presentó para los niños con TEL fue reconocimiento del título. El $75 \%$ de los niños logró completarla exitosamente. Esta tarea implica identificar el personaje principal y su acción. Esta información está explícita en el texto. En cuanto a las preguntas literales, casi un $50 \%$ de los participantes con TEL fueron capaces de responderlas. Tanto la tarea de reconocimiento del título como las preguntas literales exigen procesar la información presente en el texto. Sin embargo, el desempeño en las preguntas literales es menor al obtenido en la tarea de reconocimiento de título. Una explicación posible es que la información necesaria para responder las preguntas literales es más específica que la requerida en la tarea de reconocimiento del título. Así, dichas preguntas exigen reconocer relaciones causales, identificar distintas características de los personajes o precisar la ubicación de los personajes. Al parecer, a los niños con TEL les cuesta identificar información específica de diferente índole, aunque esté explicita en el texto.

En cuanto a la tarea identificación del resumen, 
menos del $40 \%$ es capaz de efectuarla con éxito, lo que sugiere que los niños con TEL de segundo básico aún no han desarrollado cabalmente la capacidad de construir la idea central del texto. Ello requiere procesos inferenciales, ya que la idea central no está explícita en el texto. Finalmente, las preguntas inferenciales fueron respondidas por menos del $30 \%$ de los participantes con TEL, lo que muestra que es la tarea más exigente para ellos. Los resultados relativos a la dimensión inferencial y a la tarea identificación del resumen podrían explicarse porque ambas actividades demandan relacionar información leída con el conocimiento previo, para construir representaciones mentales que den coherencia al texto (León, 2003).

Los datos correspondientes al nivel 3 sugieren que los niños con TEL tienden a presentar más comprometida la comprensión lectora que la decodificación. Esto se sustenta en el patrón de rendimiento observado en las tres subpruebas. Dicho patrón muestra que el grupo con TEL evidencia un menor rendimiento que el grupo control en las tres subpruebas. Sin embargo, en decodificación los participantes con TEL se acercaron más al desempeño del grupo control. En dos estudios previos con hablantes de lenguas transparentes se observó un fenómeno similar. En uno de ellos, en que participaron menores con TEL italianos, se determinó un incremento sustancial en el desempeño en decodificación entre primer y segundo grado (Brizzolara et al., 2006). En el otro trabajo, realizado con niños bilingües (catalánespañol) evaluados a los 8 y posteriormente a los 12 años, se estableció que se igualaron a los niños con DT en decodificación en la última evaluación. Sin embargo, mantuvieron sus diferencias en comprensión lectora (Buil-Legaz et al., 2015). El hecho de que tanto el actual trabajo como las dos investigaciones mencionadas se hayan realizado con participantes hablantes de lenguas cuya ortografía es transparente podría explicar el mejor desempeño en decodificación. Las lenguas transparentes posiblemente pueden facilitar el aprendizaje de la decodificación en los menores con TEL, debido a la consistencia de la relación entre fonema y grafema. Al respecto, existe evidencia que muestra que niños con DT hablantes de lenguas con ortografías transparentes aprenden más rápido la decodificación que los hablantes de lenguas con ortografías opacas (Caravolas \& Bruck, 1993).

El nivel 4 analiza el perfil lector de los niños con TEL. Bishop \& Snowling (2004) plantean la existencia de dificultades en decodificación y en comprensión lectora originadas por problemas en el procesamiento fonológico y en las habilidades sintácticas, semánticas y discursivas. Sin embargo, los resultados aquí presentados muestran matices. Un 44.8\% de niños con TEL no evidenció problemas lectores, un $24,1 \%$ presentó solo dificultades en comprensión lectora, otro $24,1 \%$ mostró problemas en ambas habilidades lectoras y sólo un $6.9 \%$ manifestó problemas en decodificación. Los mismos perfiles lectores fueron reportados por el estudio de Buil-Legaz et al. (2015). En su trabajo participaron 19 niños con TEL bilingües (Catalán-Español) que se distribuyeron en los siguientes desempeños lectores: 4 niños con problemas en decodificación y en comprensión lectora, 10 con sólo problemas en comprensión lectora, 1 niño evidenció sólo problemas en decodificación y 4 niños no 
presentaron problemas lectores. Tanto en este estudio como el aquí presentado se advierte un escaso número de niños con problemas referidos únicamente a la decodificación. La disminuida cantidad de niños con TEL que solo tienen problemas de decodificación apoya también la idea de que la dificultad en comprensión lectora es mayor que el problema en decodificación en ellos, al menos en hablantes de lenguas cuya ortografía es transparente.

En síntesis, el desempeño lector general de los niños con TEL de segundo básico se caracteriza por ser menor al de sus pares con DT. Los niños con TEL fueron capaces de decodificar palabras trisilábicas y comunes. En cuanto a la comprensión lectora, a nivel sintáctico comprenden oraciones. En el plano del texto logran responder apropiadamente tareas que exigen fundamentalmente identificar información literal básica, como es el personaje principal y su acción. En cambio, su desempeño es más disminuido en aquellas tareas que requieren de la elaboración de inferencias. A su vez, la mayoría de los niños con TEL evidencia dificultades en comprensión lectora, en cambio, la existencia de niños con problemas únicamente referidos a la decodificación fue muy escasa. Finalmente, los datos sugieren que la dificultad en comprensión lectora es el aspecto más comprometido en los niños con TEL.

Agradecimiento: se agradece el financiamiento otorgado por el Fondo Basal para Centros de Excelencia proyecto FB0003 de PIA-CONICYT

\section{Referencias}

Acosta, V., Moreno, A. M., \& Axpe, M. de los Á. (2010). Lenguaje oral y lectura emergente en alumnado con trastorno específico del lenguaje (TEL). Cuadernos de Comunicaçao e Linguagem, 2, 33-46.

Acosta, V., Moreno, A. M., \& Axpe, M. de los Á. (2011). Intervención sobre la conciencia fonológica en sujetos con trastorno específico del lenguaje en contextos inclusivos: posibilidades y limitaciones. Bordón. Revista de Pedagogía, 63(3), 9-22.

Acosta, V., Ramírez, G., Del Valle, N., \& de Castro, L. (2016). Intervention in reading processes in pupils with Specific Language Impairment (SLI). Psicothema, 28(1), 40-46. https://doi.org/10.7334/psicothema2015.144

Aguado, G. (2004). Trastorno específico del lenguaje. Retraso de lenguaje y disfasia. Málaga: Ediciones Aljibe.

American Speech Language Hearing Association Commitee on language, Speach and Hearing Services in the Schools. (1980). Definitions for communicative disorders on differences. ASHA, 22, 317-318.

Bishop, D., \& Adams, C. (1990). A prospective study of the relationship between specific language impairment, phonological disorders and reading retardation. Journal of Child Psychology and Psychiatry, and Allied Disciplines, 31(7), 1027-1050.

Bishop, Dorothy, McDonald, D., Bird, S., \& Hayiou-Thomas, M. (2009). Children who read words accurately despite language impairment: who are they and how do they do it? Child Development, 80(2), 593-605. https://doi.org/10.1111/j.1467-8624.2009.01281.x

Bishop, Dorothy, \& Snowling, M. (2004). Developmental dyslexia and specific language impairment: same or different? Psychological Bulletin, 130(6), 858-886. https://doi.org/10.1037/0033-2909.130.6.858

Botting, N., Simkin, Z., \& Conti-Ramsden, G. (2006). Associated Reading Skills in Children with a History of Specific Language Impairment (SLI). Reading and Writing, 19(1), 77-98. https://doi.org/10.1007/s11145-005-4322-4

Bravo, L., Villalón, M., \& Orellana, E. (2002). La Conciencia Fonológica y la Lectura Inicial en Niños que Ingresan a Primer Año Básico. Psykhe, 11(1), 175-182. https://doi.org/10.7764/psykhe.11.1.452

Brizzolara, D, Casalini, C., Gasperini, F., Roncoli, S., Mazzotti, S., Cipriani, P., \& Chilosi, A. (2006). A follow-up study of reading and writing Italian children with specific 
language impairment. En D. Riva, I. Rapin, \& G. Zardini (Eds.), Language: Normal and Pathological Development (pp. 293-252). Milano: Mariani Foundation.

Brizzolara, Daniela, Gasperini, F., Pfanner, L., Cristofani, P., Casalini, C., \& Chilosi, A. M. (2011). Long-term reading and spelling outcome in Italian adolescents with a history of specific language impairment. Cortex; a Journal Devoted to the Study of the Nervous System and Behavior, 47(8), 955973. https://doi.org/10.1016/j.cortex.2011.02.009

Buil-Legaz, L., Aguilar-Mediavilla, E., \& Rodríguez-Ferreiro, J. (2015). Reading skills in young adolescents with a history of Specific Language Impairment: The role of early semantic capacity. Journal of Communication Disorders, 58, 14-20. https://doi.org/10.1016/j.jcomdis.2015.08.001 Caravolas, M., \& Bruck, M. (1993). The Effect of Oral and Written Language Input on Children's Phonological Awareness: A Cross-Linguistic Study. Journal of Experimental Child Psychology, 55(1), 1-30. https://doi.org/10.1006/jecp.1993.1001

Carrillo, M., \& Alegría, J. (2009). Mecanismos de identificación de palabras en niños disléxicos en español: ¿Existen subtipos? Ciencias Psicológicas, 3(2), 135-152.

Catts, H., Bridges, M., Little, T., \& Tomblin, J. B. (2008). Reading achievement growth in children with language impairments. Journal of Speech, Language, and Hearing Research: JSLHR, 51(6), 1569-1579. https://doi.org/10.1044/1092-4388(2008/07-0259)

Catts, H., Fey, M., Tomblin, J. B., \& Zhang, X. (2002). A longitudinal investigation of reading outcomes in children with language impairments. Journal of Speech, Language, and Hearing Research: JSLHR, 45(6), 1142-1157.

Coloma, C. J., Pavez, M. M., Peñaloza, C., Araya, C., Maggiolo, M., \& Palma, S. (2012). Desempeño lector y narrativo en escolares con trastorno específico del lenguaje. Onomázein, (26), 351-375.

Coloma, C. J., Sotomayor, C., De Barbieri, Z., \& Silva, M. (2015). Compresión lectora, habilidades lingüísticas y decodificación en escolares con trastorno específico del lenguaje. Revista de Investigación en Logopedia, 5(1), 1-17.

De Barbieri, Z., \& Coloma, C. J. (2004). La conciencia fonológica en niños con trastorno específico de lenguaje. Revista de Logopedia, Foniatría y Audiología, 24(4), 156163. https://doi.org/10.1016/S0214-4603(04)75798-8

Defior, S., Fonseca, L., Gottheil, B., Aldrey, A., Pujals, M., Rosa, G., ... Serrano, F. (2006). LEE - Test de lectura y escritura en español. Buenos Aires: Paidós. Recuperado de http://www.leetestdelecturayescritura.com/
Diuk, B., \& Ferroni, M. (2013). ¿Anglocentrismo en los modelos de adquisición lectora? Un estudio en una lengua de ortografía transparente. Summa Psicológica UST, 10(2), 29-39.

Ehri, L. C., Nunes, S. R., Willows, D. M., Schuster, B. V., Yaghoub-Zadeh, Z., \& Shanahan, T. (2001). Phonemic Awareness Instruction Helps Children Learn to Read: Evidence from the National Reading Panel's Meta-Analysis. Reading Research Quarterly, 36(3), 250-287.

Fresneda, M. D., \& Mendoza, E. (2005). Trastorno específico del lenguaje:: concepto, clasificaciones y criterios de identificación. Revista de neurología, 41(1), 5156.

Hoover, W. A., \& Gough, P. B. (1990). The simple view of reading. Reading and Writing, 2(2), 127-160. https://doi.org/10.1007/BF00401799

Hulme, C., \& Snowling, M. J. (2011). Children's Reading Comprehension Difficulties: Nature, Causes, and Treatments. Current Directions in Psychological Science, 20(3), 139-142. https://doi.org/10.1177/0963721411408673

Kelso, K., Fletcher, J., \& Lee, P. (2007). Reading comprehension in children with specific language impairment: an examination of two subgroups. International Journal of Language \& Communication Disorders, 42(1), 39-57. https://doi.org/10.1080/13682820600693013

León, J. A. (2003). Conocimiento y discurso: claves para inferir y comprender. Madrid: Ediciones Pirámide.

Leonard, L. B. (2009). Some reflections on the study of children with specific language impairment. Child Language Teaching and Therapy, 25(2), 169-171. https://doi.org/10.1177/0265659009105891

Ministerio de Educación. Decreto Supremo 170 (2010). Recuperado https://www.leychile.cl/Navegar?idNorma=1012570

Nicolielo, A. P., \& Rocha de Vasconcellos, S. (2014). Processamento fonológico em crianças com distúrbio específico de linguagem. Revista CEFAC, 16(6), 1820-1826. https://doi.org/10.1590/1982-0216201416813

Pavez, M. M. (2003). Test exploratorio de gramática española de A. Toronto. Aplicación en Chile. Santiago, Chile: Ediciones UC. Recuperado de http://ediciones.uc.cl/ediciones-uc-educaci\%C3\%B3n-testexploratorio-gramatica-espanola-toronto-p-468.html 
Pavez, M. M. (2004). Test para la comprensión auditiva del lenguaje de E. Carrow, Aplicación en Chile. Santiago, Chile: Escuela de Fonoaudiología, Facultad de Medicina, Universidad de Chile. Recuperado de https://www.antartica.cl/antartica/servlet/LibroServlet?ac tion=fichaLibro\&id_libro $=45400$

Pavez, M. M., Maggiolo, M., \& Coloma, C. J. (2008). Test para evaluar procesos de simplificación fonológica: TEPROSIF-R. Santiago, Chile: Ediciones UC.

Ramus, F., Marshall, C. R., Rosen, S., \& van der Lely, H. K. J. (2013). Phonological deficits in specific language impairment and developmental dyslexia: towards a multidimensional model. Brain: A Journal of Neurology, 136(2), 630-645. https://doi.org/10.1093/brain/aws356

Raven, J., Court, J. H., \& Raven, C. (2005). Test de matrices progresivas. Escala coloreada, general y avanzada (1. ${ }^{\text {a }}$ ed.). Buenos Aires: Paidós. Recuperado de https://www.biblio.uade.edu.ar/client/es_ES/biblioteca/se arch/detailnonmodal;jsessionid=312C598FACF7A507E2890 8FA219C5B00?qu=PSICOLOGIA\&d=ent\%3A\%2F\%2FSD_ILS \%2F0\%2FSD_ILS\%3A317767\%7E\%7E0\%7E1\&te=ILS\&ps=30 0

Tapia, N. (2016). Desempeño lector de niños y niñas con trastorno específico del lenguaje (Tesis de Magister). Pontificia Universidad Católica de Chile, Santiago, Chile. Recuperado https://repositorio.uc.cl/handle/11534/21557

Woodcock, R., Muñoz, A., Ruef, M., \& Alvarado, C. (2005). Language survey-revised. Test Book- Spanish. Rolling Meadows: Riverside Publishing Company. 